ATZ/MTZ-Fachbuch 
Die komplexe Technik heutiger Kraftfahrzeuge und Motoren macht einen immer größer werdenden Fundus an Informationen notwendig, um die Funktion und die Arbeitsweise von Komponenten oder Systemen zu verstehen. Den raschen und sicheren Zugriff auf diese Informationen bietet die regelmäßig aktualisierte Reihe ATZ/MTZ-Fachbuch, welche die zum Verständnis erforderlichen Grundlagen, Daten und Erklärungen anschaulich, systematisch und anwendungsorientiert zusammenstellt.

Die Reihe wendet sich an Fahrzeug- und Motoreningenieure sowie Studierende, die Nachschlagebedarf haben und im Zusammenhang Fragestellungen ihres Arbeitsfeldes verstehen müssen und an Professoren und Dozenten an Universitäten und Hochschulen mit Schwerpunkt Kraftfahrzeug- und Motorentechnik. Sie liefert gleichzeitig das theoretische Rüstzeug für das Verständnis wie auch die Anwendungen, wie sie für Gutachter, Forscher und Entwicklungsingenieure in der Automobil- und Zulieferindustrie sowie bei Dienstleistern benötigt werden. 
Heiko Johannsen

\section{Unfallmechanik und Unfallrekonstruktion}

Grundlagen der Unfallaufklärung

3., überarbeitete Auflage

Mit 129 Abbildungen und 21 Tabellen

를 Springer Vieweg 
Prof. Dr.-Ing. Heiko Johannsen

Fachgebiet Kraftfahrzeuge

TU Berlin

Berlin, Deutschland

Die ersten beiden Auflagen des Buches erschienen unter dem Titel „Unfallforschung, Unfallmechanik und Unfallrekonstruktion“.

Die Deutsche Nationalbibliothek verzeichnet diese Publikation in der Deutschen Nationalbibliografie; detaillierte bibliografische Daten sind im Internet über http://dnb.d-nb.de abrufbar.

\section{Springer Vieweg}

(C) Springer Fachmedien Wiesbaden 2013

Dieses Werk einschließlich aller seiner Teile ist urheberrechtlich geschützt. Jede Verwertung, die nicht ausdrücklich vom Urheberrechtsgesetz zugelassen ist, bedarf der vorherigen Zustimmung des Verlags. Das gilt insbesondere für Vervielfältigungen, Bearbeitungen, Übersetzungen, Mikroverfilmungen und die Einspeicherung und Verarbeitung in elektronischen Systemen.

Die Wiedergabe von Gebrauchsnamen, Handelsnamen, Warenbezeichnungen usw. in diesem Werk berechtigt auch ohne besondere Kennzeichnung nicht zu der Annahme, dass solche Namen im Sinne der Warenzeichen- und Markenschutz-Gesetzgebung als frei zu betrachten wären und daher von jedermann benutzt werden dürften.

Gedruckt auf säurefreiem und chlorfrei gebleichtem Papier.

Springer Vieweg ist eine Marke von Springer DE. Springer DE ist Teil der Fachverlagsgruppe Springer Science+Business Media www.springer-vieweg.de 


\section{Vorwort zur dritten Auflage}

Dieses Buch bezieht sich auf den Pkw-Unfall im Straßenverkehr und behandelt die Themenkreise Unfallforschung, Unfallmechanik und Unfallrekonstruktion. Vorangestellt wird, um Begriffe eindeutig festzulegen, ein Kapitel zur Unfallsystematik.

Im Vordergrund stehen technische Aspekte der Unfallaufklärung. Auf medizinische und rechtliche Fragestellungen wird nur soweit wie nötig eingegangen. Zentrales Anliegen des Buches ist die Erläuterung moderner technischer Rekonstruktionsverfahren vor dem Hintergrund der klassischen Mechanik mit Impulssatz, Drehimpulssatz und Zusatzhypothesen. Grundlagen und Anwendung, Theorie und Praxis sollen verbunden, Prinzipielles und Methodisches betont werden. Die Grundlagen für das Verständnis von modernen Unfallrekonstruktionsverfahren, ob in Vorwärts- oder Rückwärtsrechnung gehalten, ob auf Stoßrechnung oder Kraftrechnung basierend, werden dargestellt und an anwendungsnahen Beispielen demonstriert.

Die technische Unfallrekonstruktion ist ein vergleichsweise junger Wissenschaftsbereich. Sein Anfang liegt in den vierziger Jahren des 20. Jahrhunderts und hat mit den Arbeiten von Brüderlin begonnen. Die stetige Weiterentwicklung der Verfahren bis in die heutige Zeit der PC-Unterstützung wird in diesem Buch nachgezeichnet.

In dieser dritten Auflage des erstmals im Jahre 1994 erschienenen Buches werden die Abschnitte Unfallsystematik, Unfallforschung und Unfallmechanik aktualisiert und der Abschnitt Unfallrekonstruktion erweitert. Definitionen werden präzisiert, Daten aktualisiert, zwischenzeitlich erschienene Veröffentlichungen und Bücher werden berücksichtigt. Behandelt werden in diesem Buch auch - nur scheinbar - nachgeordnete Fragen, über die oft hinweggegangen wird, so zum Beispiel: Wo verstecken sich bei dem grafischen Verfahren der Unfallrekonstruktion die Zusatzhypothesen? Wie ist bei rechnerischen Verfahren das Koordinatensystem zu legen? Wie unterscheidet sich die Vorzeichenfestlegung bei vorgegebenen und gesuchten Größen, z. B. Geschwindigkeiten? Warum ist zwischen eingeprägten Kräften und Reaktionskräften zu unterscheiden? Zu welcher Gruppe gehört die Stoßkraft bzw. der Stoßantrieb, welche Konsequenzen ergeben sich daraus?

Das Buch basiert auf langjähriger Forschung und Lehre im Fachgebiet Kraftfahrzeuge der Technischen Universität Berlin.

Das Buch wendet sich vornehmlich an Unfall- und Kraftfahrzeug-Sachverständige, seien sie selbstständig oder für Versicherungen bzw. Überwachungsvereine tätig. Gerade dem 
erfahrenen, in der Praxis tätigen Unfallgutachter soll mit dem Buch die Rückbesinnung auf die physikalischen Grundlagen der von ihm entweder explizit oder, in Software eingebunden, implizit angewandten Verfahren erleichtert werden. Darüber hinaus möge das Buch den Studenten an Universitäten und Hochschulen bei ihrer Ausbildung behilflich sein. Das Buch eignet sich hier als Grundlage und Leitfaden bei Vorlesungen und Übungen. Auch den Sicherheitsingenieuren bei Automobilherstellern und Sicherheitszulieferern wird das Buch nützlich sein. Schließlich soll das Buch jungen Ingenieuren den Einstieg in die technische Unfallrekonstruktion, Unfallanalyse und Unfallforschung erleichtern und deren Verbindungen zur Sicherheitsforschung aufzeigen.

Diese Buch basiert im Wesentlichen auf der 2. Auflage von Professor Dr.-Ing. Hermann Appel, Dr.-Ing. Gerald Krabbel und Dirk Vetter. Bei der vorliegenden Überarbeitung und Neugestaltung des Buches hat Herr cand. ing. T. Berger tatkräftig mitgeholfen. Des Weiteren basiert die Überarbeitung teilweise auf fruchtbaren Diskussionen mit Gerd Müller und Mathias Stein. Wesentliche Teile der In-Depth Datenanalysen wurden freundlicher Weise von Prof. Dietmar Otte bereitgestellt. Ihnen allen habe ich sehr zu danken.

Berlin, im Juni 2013

Heiko Johannsen 


\section{Inhaltsverzeichnis}

Abkürzungen, Bezeichnungen, Formelzeichen $\ldots \ldots \ldots \ldots \ldots \ldots \ldots$

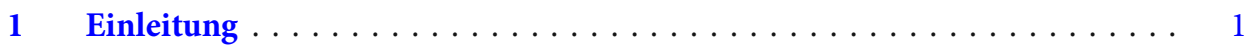

2 Unfallsystematik ....................... 5

2.1 Definitionen: Unfall, Risiko, Sicherheit . . . . . . . . . . . . 5

2.2 Zeitliche Phasen des Unfalls . . . . . . . . . . . . . . . . 11

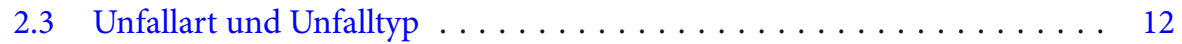

2.4 Kollisionsart und Kollisionstyp . . . . . . . . . . . . . . . . . 17

2.5 Aufprallart und Aufpralltyp . . . . . . . . . . . . . . . . . . 20

2.6 Belastungs- und Verletzungsarten des Menschen . . . . . . . . . . . . . . 25

3 Unfallforschung . . . . . . . . . . . . . . . . . 27

3.1 Bearbeitungskette von der Unfallerhebung bis zur Unfallforschung . . . 27

3.2 Unfallerhebungen . . . . . . . . . . . . . . . . . 28

3.2.1 Polizeiliche Unfallerhebungen . . . . . . . . . . . . . . 31

3.2.2 Erhebungen am Unfallort durch spezielle Forschungsteams . . . . 32

3.2.3 Retrospektive Unfalluntersuchungen . . . . . . . . . . . 36

3.3 Unfallrekonstruktion und Unfallaufklärung, Rechtsprechung . . . . . . . 41

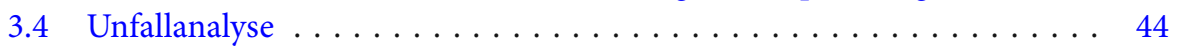

3.5 Unfallschwere, Unfallfolgenschwere . . . . . . . . . . . . . . . . . . 46

3.5.1 Unfallschwere, Unfallkenngröße . . . . . . . . . . . . . . 47

3.5.2 Unfallfolgen, Unfallfolgenschwere . . . . . . . . . . . . . 52

3.6 Unfallforschung, Sicherheitsforschung . . . . . . . . . . . . . . 57

3.7 Ausgewählte Ergebnisse der Unfallanalyse, Unfallforschung und Sicherheitsforschung . . . . . . . . . . . . . . . . 59

$4 \quad$ Unfallmechanik . . . . . . . . . . . . . . . . . . 85

4.1 Kinematische Grundlagen . . . . . . . . . . . . . . . 85

4.2 Crashkinematik, Crashpulse, Stoßziffer (Stoffgesetze) . . . . . . . . . 88

4.2.1 Crashkinematik, Deformationskennungen . . . . . . . . . 88

4.2 .2 Stoßziffer . . . . . . . . . . . . . . . . . . 95 
4.3 Kraftrechnung ....................... 97

4.3 .1 Schwerpunktsatz . . . . . . . . . . . . . . . . . . 99

4.3 .2 Drallsatz . . . . . . . . . . . . . . . . 100

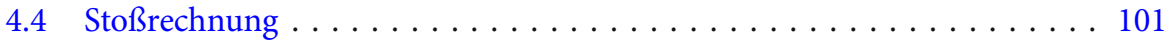

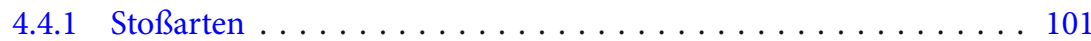

4.4 .2 Stoßtheorien . . . . . . . . . . . . . . . . . 102

4.4 .3 Ergänzungshypothesen . . . . . . . . . . . . . . . . . 104

4.4 .4 Impulssatz . . . . . . . . . . . . . . . . . . . 105

4.4.5 Drehimpulssatz . . . . . . . . . . . . . . . . . . . . 105

4.4.6 Stoßgleichungen für die ebene Scheibe . . . . . . . . . . . . . 106

4.4.7 Gerader zentrischer Stoß zweier Scheiben . . . . . . . . . . . . . . 107

4.4.8 Schiefer exzentrischer Stoß zweier Scheiben . . . . . . . . . . . . 110

4.5 Arbeitssatz (Energiesatz) . . . . . . . . . . . . . . . . . . . 112

4.5.1 Arbeitssatz für starre Körper . . . . . . . . . . . . . . . . . . . 112

4.5.2 Arbeitssatz für deformierbare Körper im statischen Gleichgewicht 114

4.5.3 Arbeitssatz allgemein . . . . . . . . . . . . . . . . . . 115

4.5.4 Arbeitssatz für den Wandaufprall eines Fahrzeugs . . . . . . . . . 115

4.5.5 Arbeitssatz für die gerade, zentrische Kollision zweier Fahrzeuge . 116

4.5.6 Arbeitssatz für den exzentrischen Aufprall eines Fahrzeugs auf einen starren Pfahl . . . . . . . . . . . . . . . . . 117

4.5.7 Arbeitssatz für die Auslaufbewegung nach dem Stoß . . . . . . . . 117

4.5.8 Berechnung der Stoßziffer für einen Wandaufprall . . . . . . . . . . . 119

4.6 Anwendungsbeispiele zur Kraftrechnung . . . . . . . . . . . . . . . . . 120

4.6.1 Physikalisches Pendel. . . . . . . . . . . . . . . . . . 120

4.6.2 Frontalkollision zweier Fahrzeuge . . . . . . . . . . . . . . 121

4.7 Anwendungsbeispiele zur Stoßrechnung . . . . . . . . . . . . . . . . . 125

4.7.1 Exzentrischer Stoß gegen eine drehbar gelagerte Scheibe . . . . . . 125

4.7.2 Kollision Pkw-Baum . . . . . . . . . . . . . . . . . 128

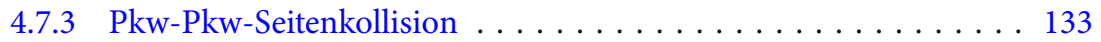

4.7.4 Pkw-Pkw-Offset-Frontalkollision . . . . . . . . . . . . 135

4.7.5 Kollision Pkw-Fußgänger . . . . . . . . . . . . . . . . . 138

4.7.6 Frontalaufprall ohne und mit Abgleiten,

Einfluss auf Geschwindigkeitsänderung und Energieabsorption . . 144

4.8 Festlegung der Unbekannten in Ersatzsystemen . . . . . . . . . . . . . . . 146

5 Unfallrekonstruktion . . . . . . . . . . . . . . . . . . . . . . . . . . . . . . . . . 149

5.1 Unfallrekonstruktionsverfahren . . . . . . . . . . . . . . . . . . 150

5.1.1 Rückwärts-Unfallrekonstruktion, insbesondere Rückwärts-Kollisionsrekonstruktion . . . . . . . . 153

5.1.2 Vorwärts-Unfallrekonstruktion, insbesondere Vorwärts-Kollisionsrekonstruktion . . . . . . . . 156

5.1 .3 Kontrollmöglichkeiten . . . . . . . . . . . . . . . . . . . . 159 
5.1 .4 Unfalldatenspeicher . . . . . . . . . . . . . . . . . . . 160

5.2 Grafische Rekonstruktionsverfahren . . . . . . . . . . . . . . . . . . 162

5.2 .1 Antriebs-Balance-Verfahren . . . . . . . . . . . . . . 163

5.2 .2 Impuls-Spiegelverfahren . . . . . . . . . . . . . . . . . . . . 165

5.2 .3 Drehimpuls-Spiegelverfahren . . . . . . . . . . . . . . . . 167

5.2.4 Energie-Ringverfahren . . . . . . . . . . . . . . . . . . . . . . 169

5.2.5 Rhomboid-Schnittverfahren . . . . . . . . . . . . . . . . . 172

5.2.6 Band-Schnittverfahren . . . . . . . . . . . . . . . . 173

5.3 Rechnerische Rekonstruktionsverfahren . . . . . . . . . . . . . . . . 177

5.4 Anmerkungen und Ergänzungen

zu den vorgestellten Rekonstruktionsverfahren . . . . . . . . . . . . . . 179

5.5 Einflüsse auf die Qualität von Rekonstruktionsergebnissen . . . . . . . . . 182

Anhang ........................... 187

Literatur . . . . . . . . . . . . . . . . . . . . . . . . 199

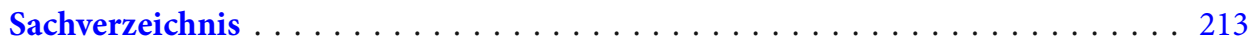




\section{Abkürzungen, Bezeichnungen, Formelzeichen}

\section{Abkürzungen}

$\begin{array}{ll}\text { ABS } & \text { Anti-Blockiersystem (Bezeichnung der Fa. Bosch für ihre ABV) } \\ \text { ABV } & \text { Automatischer Blockierverhinderer } \\ \text { ACC } & \text { Autonomous Cruise Control (oder Adaptive Cruise Control) } \\ \text { ACIR } & \text { Automotive Crash Injury Research (USA) } \\ \text { ADAC } & \text { Allgemeiner Deutscher Automobilclub e. V. } \\ \text { ADR } & \text { Australian Design Rules } \\ \text { AIS } & \text { Abbreviated Injury Scale alt.: Automotive Industry Standards (Indien) } \\ \text { ANCAP } & \text { Australian New Car Assessment Programme } \\ \text { APF } & \text { Abdominal Peak Force } \\ \text { BASt } & \text { Bundesanstalt für Straßenwesen } \\ \text { BMFT } & \text { Bundesministerium für Forschung und Technologie, jetzt Bundesministeri- } \\ & \text { um für Bildung und Forschung (BMBF) } \\ \text { BMW } & \text { Bayrische Motorenwerke AG } \\ \text { BUB } & \text { bewegliche undeformierbare Barriere } \\ \text { C-NCAP } & \text { China New Car Assessment Programme } \\ \text { CARAT } & \text { Computer Aided Reconstruction of Accidents in Traffic } \\ \text { CDC } & \text { Collision Deformation Classification } \\ \text { CDS } & \text { Crashworthiness Data System (Unterprogramm von NASS, sammelt statisti- } \\ & \text { sche Daten zu Fahrzeugkollisionen) } \\ \text { CRASH } & \text { Calspan Reconstruction of Accident Speeds on the Highway } \\ \text { DAT } & \text { Deutsche Automobil Treuhand GmbH } \\ \text { DEKRA } & \text { Deutscher Kraftfahrzeug-Überwachungs-Verein e. V. } \\ \text { DIN } & \text { Deutsches Institut für Normung e. V. } \\ \text { DOT } & \text { Department of Transportation (USA) } \\ \text { Dummy } & \text { Messpuppe } \\ \text { EBS } & \text { Equivalent Barrier Speed (veraltet) } \\ \text { ECE } & \text { Economic Commission of Europe } \\ & \end{array}$


EDCRASH Engineering Dynamics Corporation Reconstruction of Accident Speeds on the Highway, Unfallrekonstruktionsprogramm der Engineering Dynamics Corporation (USA)

EES Equivalent Energy Speed

ESP Elektronisches Stabilitätsprogramm

ESV Experimental Safety Vehicle

ETS Equivalent Test Speed

Euro NCAP European New Car Assessment Programme

EVU Europäischer Verein für Unfallforschung und Unfallanalyse e. V.

FARS Fatality Analysis Reporting System (USA)

FAT Forschungsvereinigung Automobiltechnik e. V. im VDA

FEM Finite Elemente Methode

Fg Fußgänger

FMVSS Federal Motor Vehicle Safety Standard

FOLKSAM Automobilversicherer in Schweden

Fr Fahrrad, Fahrradfahrer

GB Guobiao (nationale Norm in China)

GDV Gesamtverband der Deutschen Versicherungswirtschaft e. V.

GIDAS German In Depth Accident Study

GTÜ Gesellschaft für Technische Überprüfungen

GUVU Gesellschaft für Ursachenforschung bei Verkehrsunfällen e. V.

HIC Head Injury Criterion

HLDI Highway Loss Data Institute (USA, Institut der Versicherungswirtschaft)

HPC Head Protection Criterion (wie HIC, aber Untersuchungszeit begrenzt auf Kontaktzeitraum)

HUK Verband der Haftpflichtversicherer, Unfallversicherer, Autoversicherer und Rechtsschutzversicherer e. V. (HUK-Verband), München. Abgelöst durch GDV

IIHS Insurance Institute for Highway Safety (USA)

INRETS Institut National De Recherche Sur Les Transports Et Leur Sécurité (Frankreich), heute IFSTTAR

IR Infrarot

IRCOBI International Research Committee On Biomechanics

ISS Injury Severity Score

JNCAP Japan New Car Assessment Programme

Kfz (KFZ) Kraftfahrzeug

KMVSS Korean Motor Vehicle Safety Standard (Südkorea)

KNCAP Korean New Car Assessment Programme

KT Kollisionstyp

Latin NCAP Latin New Car Assessment Programme (Südamerika)

LV Leicht Verletzte

M Momentanpol, Drehpol 
MAIS Maximum AIS = Maximaler AIS-Wert bei einem Verletzten

MB Mercedes Benz

MHH Medizinische Hochschule Hannover

MKS Mehrkörpersystem

$\mathrm{Mz} \quad$ Motorisierte Zweiradfahrer

NASS National Automotive Sampling System (USA, 1966 eingeführt)

NCAP New Car Assessment Program (USA)

NCSA National Center for Statistics and Analysis (USA), „Statistisches Bundesamt“ der USA, bei dem z. B. die Unfallerhebungsdaten aus NASS einfließen

Nfz (NFZ) Nutzkraftwagen

NHTSA National Highway Traffic Safety Administration (USA), amerikanische Verkehrssicherheitsbehörde im DOT

NIC Neck Injury Criterion

Nij Normalised Neck Injury Criterion

ODB ortsfeste deformierbare Barriere

OOP Out of Position

OUB ortsfeste undeformierbare Barriere

OWiG Ordnungswidrigkeitengesetz

Pkw (PKW) Personenkraftwagen

PMTO Post Mortal Testobject (Leiche)

PODS Probability of Death Score

PSPF Pubic Symphysis Peak Force

RDC Rib Deflection Criterion

S Schwerpunkt

SAE Society of Automotive Engineers, Inc., Warrendale, DA, USA

SID Side Impact Dummy

SNPRM Supplemental Notice of Proposed Rulemaking

SPUL Spezifische Unfall-Leistung

STAPP Name, daher Stapp Conference

StPO Strafprozessordnung

SV schwer Verletzte

TRIAS Type Approval Test Procedures (Japan)

TRL Transport Research Laboratory (England), früher TRRL

TRRL Transport Road Research Laboratory (England)

TTI Thoracic Trauma Index

TUB Technische Universität Berlin

TUD Technische Universität Dresden

TÜV Technischer Überwachungsverein

TV tödlich Verletzte

UDS Unfall-Daten-Speicher (früher: Unfall-Daten-Schreiber)

US-NCAP US New Car Assessment Programme

VC Viscous Criterion 
VDA Verband der Automobilindustrie e. V.

VDI Vehicle Deformation Index

VDI Verein Deutscher Ingenieure

VIDI Vehicle Interior Deformation Index (inzwischen nicht mehr angewandt)

VOI Volkswirtschaftlich Optimierter Insassenschutz (BMFT-Forschungsprojekt)

VTI Swedish National Road and Transport Research Institute

VW Volkswagen AG

\section{Formelzeichen, Bezeichnungen}

\begin{tabular}{|c|c|c|}
\hline$\alpha$ & & Einlaufwinkel \\
\hline$\mu$ & & Reibwert \\
\hline$\varphi$ & Bog & Drehwinkel \\
\hline$\omega$ & $\mathrm{Bog} / \mathrm{s}$ & Winkelgeschwindigkeit \\
\hline$\Theta$ & $\mathrm{kgm}^{2}$ & Massenträgheitsmoment \\
\hline$\Theta_{0}$ & $\mathrm{kgm}^{2}$ & Massen-Trägheitsmoment bezogen auf den Punkt 0 \\
\hline$\stackrel{\Theta_{0}}{=}$ & $\mathrm{kgm}^{2}$ & Massen-Trägheitsmatrix bezogen auf den Punkt 0 \\
\hline $\bar{a}$ & & Index für äußere Kraft \\
\hline$\underline{a}$ & $\mathrm{~m}$ & Abstandsvektor des Stoßantriebes \\
\hline$a$ & $\mathrm{~m} / \mathrm{s}^{2}$ & Beschleunigung (Betrag) \\
\hline$A$ & $\mathrm{Nm}$ & Arbeit \\
\hline$A, E$ & & Anfang, Ende einer Bewegung \\
\hline$B$ & & $\begin{array}{l}\text { Berührungspunkt, Kontaktpunkt, Impulsübertragungspunkt am } \\
\text { Fahrzeug (identisch mit } K \text { ) }\end{array}$ \\
\hline$c$ & $\mathrm{~N} / \mathrm{m}$ & Steifigkeit \\
\hline$c_{\text {ers }}$ & $\mathrm{N} / \mathrm{m}$ & Ersatzsteifigkeit \\
\hline$D$ & $\mathrm{kgm}^{2}$ & Drall \\
\hline$D$ & $\mathrm{~m}$ & Eindrückung \\
\hline $1 \mathrm{D}, 2 \mathrm{D}, 3 \mathrm{D}$ & & ein-, zwei-, dreidimensional \\
\hline$\Delta v$ & $\mathrm{~m} / \mathrm{s}$ & Geschwindigkeitsänderung \\
\hline$e$ & & Index für eingeprägte Kraft \\
\hline$\underline{e}$ & & Einheitsvektor \\
\hline$\underline{e}$ & $\mathrm{~m}$ & minimaler Abstandsvektor des Stoßantriebes \\
\hline E & $\mathrm{Nm}$ & Kinetische Energie \\
\hline EES & $\mathrm{km} / \mathrm{h}$ & Equivalent Energy Speed \\
\hline$F$ & $\mathrm{~N}$ & Kraft \\
\hline$h$ & $\mathrm{~m}$ & Höhe \\
\hline$i$ & & Index für innere Kraft \\
\hline$i$ & $\mathrm{~m}$ & Trägheitsradius \\
\hline$I, \underline{I}$ & $\mathrm{kgm} / \mathrm{s}, \mathrm{Ns}$ & Impuls, Impulsvektor \\
\hline
\end{tabular}




\begin{tabular}{|c|c|c|}
\hline \multicolumn{2}{|l|}{$k$} & Restitutionskoeffizient, Stoßziffer, Stoßzahl \\
\hline \multicolumn{2}{|l|}{ K } & $\begin{array}{l}\text { Berührungspunkt, Kontaktpunkt, Impulsübertragungspunkt am } \\
\text { Fahrzeug (identisch mit } B \text { ) }\end{array}$ \\
\hline KO & & Kollisionsort auf der Straße \\
\hline$l$ & $\mathrm{~m}$ & Länge \\
\hline$l_{\mathrm{R}}, R$ & $\mathrm{~m}$ & Radstand \\
\hline$m$ & $\mathrm{~kg}$ & Masse \\
\hline$M$ & $\mathrm{Nm}$ & Moment \\
\hline$M$ & & Momentanpol \\
\hline$M_{\mathrm{D}}$ & & Drehpol \\
\hline$m_{\mathrm{ers}}$ & $\mathrm{kg}$ & Ersatzmasse \\
\hline$N$ & & Normalrichtung des Stoßantriebes \\
\hline$N$ & $\mathrm{kgm} / \mathrm{s}^{2}$ & Newton \\
\hline$P, P$ & $\mathrm{kgm} / \mathrm{s}, \mathrm{Ns}$ & Stoßantrieb, Stoßantriebsvektor \\
\hline$P^{\star}$ & $\mathrm{kgm} / \mathrm{s}, \mathrm{N} \mathrm{s}$ & kleinstmöglicher Stoßantrieb, Hilfsgröße \\
\hline$\underline{r}$ & $\mathrm{~m}$ & Lagevektor \\
\hline$R$ & & Index für Reaktionskraft \\
\hline$R$ & $\mathrm{~m}$ & Radstand \\
\hline$s$ & $\mathrm{~m}$ & Weglänge, Deformationsweg \\
\hline $\mathrm{s}$ & & Sekunde \\
\hline$S$ & & Schwerpunkt \\
\hline SPUL & $\mathrm{m}^{2} / \mathrm{s}^{2}$ & Spezifische Unfallleistung \\
\hline$T$ & & Tangentialrichtung des Stoßantriebes \\
\hline$T$ & $\mathrm{~s}$ & Vollschwingungsdauer \\
\hline$t$ & $\mathrm{~s}$ & Zeit \\
\hline$v$ & $\mathrm{~m} / \mathrm{s}$ & Geschwindigkeit \\
\hline$v_{\mathrm{koll}}, v_{0}$ & $\mathrm{~m} / \mathrm{s}$ & Aufprall-, Kollisionsgeschwindigkeit \\
\hline$v_{\text {rel }}$ & $\mathrm{m} / \mathrm{s}^{2}$ & Relativgeschwindigkeit \\
\hline$W, W_{\text {Def }}$ & $\mathrm{Nm}$ & Deformationsarbeit \\
\hline$W_{\mathrm{el}}$ & $\mathrm{Nm}$ & elastische Deformationsarbeit \\
\hline$W_{\mathrm{pl}}$ & $\mathrm{Nm}$ & plastische Deformationsarbeit \\
\hline$x$ & & äußeres Produkt von Vektoren \\
\hline$x, y, z$ & $\mathrm{~m}$ & Lagekoordinaten (i. Allg. für den Schwerpunkt) \\
\hline$x(t)$ & $\mathrm{m}$ & zeitabhängiger Weg \\
\hline$\dot{x}(t)$ & $\mathrm{m} / \mathrm{s}$ & zeitabhängige Geschwindigkeit \\
\hline$\ddot{x}(t)$ & $\mathrm{m} / \mathrm{s}^{2}$ & zeitabhängige Beschleunigung \\
\hline()$,()^{\prime}$ & & vor, nach dem Stoß \\
\hline$\left(\_\right)$ & & einfache Unterstreichung für Vektor \\
\hline$(=)$ & & doppelte Unterstreichung für Matrix, Tensor \\
\hline
\end{tabular}

